\title{
Estratégias de aprendizagem no ensino remoto: Um estudo com licenciandos em
}

\section{Química e Biologia}

\author{
Learning strategies in remote education: A study with undergraduate students in chemistry and \\ biology
}

Estrategias de aprendizaje en educación remota: Un estudio con estudiantes de grado en química y biologia

\author{
Shirley dos Santos Dias \\ ORCID: https://orcid.org/0000-0001-9812-3452 \\ Universidade Federal do Amazonas, Brasil \\ E-mail: shirleymoreira192@gmail.com \\ Jean Michel dos Santos Menezes \\ ORCID: https://orcid.org/0000-0003-2769-1887 \\ Universidade Federal do Amazonas, Brasil \\ E-mail: jeanmichelsm@ufam.edu.br \\ Priscila Duarte de Lira \\ ORCID: https://orcid.org/0000-0002-9081-853X \\ Universidade Federal do Amazonas, Brasil \\ E-mail: pridlira@gmail.com \\ Dominique Fernandes de Moura do Carmo \\ ORCID: https://orcid.org/0000-0002-8835-1619 \\ Universidade Federal do Amazonas, Brasil \\ E-mail: dominiquefmc@ufam.edu.br
}

\begin{abstract}
Resumo
O ensino remoto, apoiado por mídias e tecnologias vem crescendo no Brasil. O contexto pandêmico fez com que essa modalidade de ensino fosse adotada de maneira emergencial. Grande parte dos estudantes não está acostumada a estudar de maneira não presencial, o que pode interferir no seu processo de aprendizagem. Dessa forma, objetivou-se identificar as estratégias de aprendizagem utilizadas por estudantes de licenciatura em Química e Biologia durantes as aulas remotas emergenciais em uma disciplina de Química Geral. A coleta de dados se deu por meio de um questionário em escala Likert. Os dados foram tratados quantitativamente e analisados qualitativamente. Os resultados mostram que as estratégias de aprendizagem cognitivas mais utilizadas pelos licenciandos se voltavam a ação de anotar o que eles consideram importante em determinado assunto. Em relação as estratégias metacognitivas (mais usadas pelos participantes), as mais frequentes foram aquelas voltadas a ler e reler textos com atenção, tentando superar a dificuldade ou para ter certeza de que estão compreendendo aquele conteúdo.
\end{abstract}

Palavras-chave: Estratégias de aprendizagem; Ensino remoto; Ensino de ciências.

\begin{abstract}
Remote learning, supported by media and technologies, has been growing in Brazil. The pandemic context made this teaching modality to be adopted as an emergency. Most students are not used to studying in person, which can interfere with their learning process. Thus, the objective was to identify the learning strategies used by undergraduate students in Chemistry and Biology during emergency remote classes in a General Chemistry course. Data collection took place through a Likert scale questionnaire. Data were treated quantitatively and analyzed qualitatively. The results show that the cognitive learning strategies most used by undergraduates were focused on writing down what they consider important in a given subject. Regarding metacognitive strategies (most used by participants), the most frequent were those aimed at reading and rereading texts carefully, trying to overcome the difficulty or to make sure they understand that content.
\end{abstract}

Keywords: Learning strategies; Remote education; Science teaching.

\section{Resumen}

El aprendizaje remoto, apoyado por medios y tecnologías, ha ido creciendo en Brasil. El contexto de la pandemia hizo que esta modalidad de enseñanza fuera adoptada como una emergencia. La mayoría de los estudiantes no están acostumbrados a estudiar en persona, lo que puede interferir con su proceso de aprendizaje. Así, el objetivo fue identificar las estrategias de aprendizaje utilizadas por los estudiantes de pregrado en Química y Biología durante las clases remotas de emergencia en una disciplina de Química General. La recogida de datos se realizó mediante un 
cuestionario en escala Likert. Los datos se trataron cuantitativamente y se analizaron cualitativamente. Los resultados muestran que las estrategias de aprendizaje cognitivo más utilizadas por los estudiantes de pregrado se centraron en anotar lo que consideran importante en una materia determinada. En cuanto a las estrategias metacognitivas (más utilizadas por los participantes), las más frecuentes fueron las dirigidas a leer y releer textos con atención, intentando superar la dificultad o asegurarse de que comprenden ese contenido.

Palabras clave: Estrategias de aprendizaje; Educación remota; Enseñanza de la ciencia.

\section{Introdução}

Vivemos hoje em uma sociedade complexa, inundada por fontes de informação. A tecnologia digital vem alterando os processos de aprendizagem das novas gerações, porém, precisa ser explorada a partir de pressupostos pedagógicos que a reconhecem como agência de habilidades cognitivas e formas de aprender. O mundo, marcado por tanta riqueza informativa e avanços tecnológicos, precisa urgentemente do poder esclarecedor do pensamento (Bannel et al., 2016).

Filatro (2018, p. 52) define tecnologia como o "veículo usado para comunicar informações que estão representadas em uma ou mais mídias". No campo educacional, a tecnologia abrange o conjunto de ferramentas ou serviços usados para apoiar o ensino e a aprendizagem, viabilizando a realização de atividades.

Com o crescimento do acesso da população aos dispositivos móveis, como smartphones e computadores, essas ferramentas usadas em ambientes virtuais de aprendizagem se tornou uma opção válida e eficaz na educação (Moreira et al., 2020).

Desse modo, os ambientes educacionais enquanto espaço de formação precisam compreender, integrar e aperfeiçoar o uso dessas tecnologias digitais ao processo de ensino e aprendizagem sempre que possível, uma vez que a sociedade atual caminha para um cenário mais tecnológico, como o ensino remoto (Vieira, et al., 2019).

No ensino remoto, apoiado por mídias e tecnologias, quase toda interação do estudante com a proposta educacional se inicia a partir dos conteúdos. Por isso, para preparar esses conteúdos é necessário incorporar nos materiais digitais boa parte da comunicação didática que acontece na educação presencial (Filatro, 2018).

O reflexo das tecnologias digitais na educação e a crescente adoção do ensino remoto revelam a importância do uso de novas metodologias e estratégias de ensino e aprendizagem que possam auxiliar o êxito no processo educacional. Com isso, além de mudar culturalmente o que se aprende, muda-se também como se aprende, requerendo-se novas abordagens de ensino e de aprendizagem (Hargreaves, 2004; Horowitz et al., 2019).

Piletti (2004) define "metodologia" no campo educacional como um conjunto de procedimentos didáticos expressados por seus métodos e técnicas de ensino logicamente coordenados que guiam a aprendizagem dos alunos para determinados fins. Já o termo "estratégia" é definido por Stuart (2010) como procedimentos, recursos e ações utilizadas no processo educativo para promover a aprendizagem significativa de forma intencional para que o aluno processe o conteúdo de forma profunda e consciente. A estratégia pode ocorrer e se apresentar de formas diferentes ao longo do processo.

Estratégias de aprendizagem podem ser definidas como sequências de procedimentos que se escolhem com o propósito de facilitar a aquisição, o armazenamento e a utilização de uma informação, sendo esses procedimentos conscientes e intencionais. As estratégias podem ser modificadas por ações educacionais com o intuito de aumentar o ganho de aprendizagem em uma atividade ou ambiente específico (Zerbini \& Abbad, 2008)

Essas estratégias são classificadas, de uma maneira geral, em cognitivas ou metacognitivas. As estratégias cognitivas estão relacionadas a comportamentos e pensamentos que influenciam o processo de aprendizagem, de maneira que a informação possa ser armazenada com mais eficiência (por exemplo, sublinhar, anotar). Tais estratégias estão relacionadas ao fato de o indivíduo perceber analiticamente as partes visando entender o todo. Por sua vez, as estratégias metacognitivas se referem a procedimentos que o aluno adota para planejar, monitorar e regular o seu próprio pensamento, e têm a função de levá-lo à reflexão acerca de sua própria aprendizagem, permitindo a busca de novas soluções para aprender de modo a 
conseguir superar os obstáculos. Estas estratégias estão presentes quando o sujeito identifica qual a melhor maneira de resolver uma situação-problema proposta, por exemplo (Oliveira et al., 2010; Boruchovitch \& Gomes, 2019).

Os estudantes que recorrem com mais frequência a estratégias metacognitivas tendem a ter melhor rendimento escolar na disciplina em que essa estratégia ocorre. Essas estratégias de aprendizagem podem ser ensinadas para alunos com o intuito de melhorar o desempenho nas aulas (Rosa, 2011; Castro et al., 2016; Andrade \& Zerbini, 2020).

Nesse contexto, devido a pandemia do Covid-19 e respeitando as orientações das organizações de saúde para adoção de medidas de distanciamento social, as instituições de ensino tiveram que se adaptar a uma nova rotina e adotaram o ensino remoto de maneira emergencial para desenvolver suas atividades. Assim, é importante que os alunos compreendam as diferenças existentes entre o ensino presencial e o ensino remoto, adotando estratégias que os auxiliem a aprender nessa modalidade.

A adaptação do aluno ao ensino apoiado por mídias digitais é uma variável relevante para entender o comportamento do universitário no decorrer de sua formação. Dessa forma, objetivou-se identificar as estratégias de aprendizagem utilizadas por estudantes de licenciatura em Química e Biologia durantes as aulas remotas.

\section{Metodologia}

O estudo apresenta caráter predominantemente qualitativo. A pesquisa qualitativa considera a existência de uma relação dinâmica entre o mundo real e o sujeito. É descritiva, interpretativa, utiliza o método indutivo e foca principalmente no processo e nas perspectivas dos atores sociais envolvidos, como professores, alunos etc. Em educação, essa abordagem utiliza principalmente métodos que possibilitam a obtenção de dados descritivos que permitem observar o modo de pensar dos indivíduos pesquisados (Massoni, 2016). Por mais que o instrumento se utilize de um tratamento quantitativo dos dados, a discussão será realizada por uma abordagem qualitativa.

A pesquisa foi realizada com 14 estudantes do curso de Licenciatura em Ciências: Química e Biologia, ofertado pelo Instituto de Ciências Exatas e Tecnologia, unidade da Universidade Federal do Amazonas (UFAM) localizada no município de Itacoatiara/AM.

Esse curso de licenciatura dupla foi criado em novembro de 2005 com o objetivo de atender a demanda da Educação Básica local, desta forma, os professores formados possuem amplo domínio dos conhecimentos pertinentes ao ensino em Ciências: Química e Biologia, aptos a empregar esses conhecimentos com adequação pedagógica.

Os licenciandos estavam cursando a disciplina Química Geral I, sendo ministrada por meio de plataformas digitais. Esses participantes responderam, por meio de formulário eletrônico, a um questionário adaptado do trabalho de Boruchovitch (2006) e Oliveira et al. (2009), composto por 40 itens que avaliam as estratégias empregadas por alunos em situação de aprendizagem e identifica o tipo de estratégia utilizada. $\mathrm{O}$ instrumento de coleta de dados apresentou uma escala com três pontos, com características de respostas do tipo Likert, sendo "nunca", "às vezes" e "sempre".

O método de escala Likert foi desenvolvido por Rensis Liket em 1932 e é definido como um conjunto de itens apresentados como afirmações ou opiniões, para o qual se pede a reação dos participantes. Ou seja, a cada afirmação é solicitado que o respondente manifeste sua reação escolhendo um dos pontos ou categorias da escala. Para cada ponto atribuise um valor numérico (Sampieri et al., 2013). A pontuação das respostas para o questionário aplicado foi: 1 ponto para "nunca", 2 pontos para "às vezes" e 3 pontos para "sempre".

O tratamento quantitativo dos dados foi realizado por meio da estatística descritiva. Esse tipo de estatística pode ser considerado como um conjunto de técnicas analíticas utilizado para resumir dados recolhidos em uma pesquisa, que são geralmente organizados em tabelas e gráficos, evidenciando valor mínimo e máximo, soma dos valores, dentre outros (Huot, 2002). 
Assim, foram determinadas por afirmativa: a Pontuação Máxima (PMáx) e Pontuação Mínima (PMin) da escala; a Amplitude (A), que é a diferença entre a PMáx e a Pmin, e indica o número de unidades na escala de mensuração que precisamos para incluir o valor máximo e mínimo; o Ranking Médio (RM), que é a medida de tendência central mais utilizada; e a Pontuação Total (PT). Para a interpretação dos valores encontrados, considera-se que quanto mais próximo de 3 o RM estiver maior será frequência que os participantes utilizam determinada estratégia e quanto mais próximo de 1 menor será. O tratamento dos dados foi realizado no programa Microsoft Excel.

\section{Resultados e Discussão}

Por meio das respostas dos licenciandos foi possível identificar algumas estratégias de aprendizagem que são adotadas por eles, bem como a frequência que eles as utilizam. Os estudantes apresentaram ser relativamente bons usuários de estratégias de aprendizagem, porém, reportaram menor frequência de uso das estratégias do tipo cognitivas (Tabela 1), quando comparadas às metacognitivas.

Tabela 1. Dados referentes as estratégias de aprendizagem cognitivas.

\begin{tabular}{|c|c|c|c|c|c|c|}
\hline Item & Afirmativa & Pmáx & Pmin & $\mathbf{A}$ & MA & PT \\
\hline 3 & Cria perguntas e respostas sobre o assunto que está estudando & 3 & 1 & 2 & 1,79 & 25 \\
\hline 4 & Organiza um roteiro para estudar & 3 & 1 & 2 & 1,86 & 26 \\
\hline 5 & Repete as atividades do curso em casa para treinar & 3 & 1 & 2 & 2,29 & 32 \\
\hline 6 & Faz uma lista de ideias antes de começar a escrever um texto & 3 & 1 & 2 & 2,21 & 31 \\
\hline 7 & Procura reescrever um texto com suas palavras para estudar depois & 3 & 1 & 2 & 2,21 & 31 \\
\hline 12 & Faz desenhos ou esquemas para entender melhor o que está sendo estudado & 3 & 1 & 2 & 1,93 & 27 \\
\hline 13 & Usa o dicionário ao escrever um texto (resumo, e-mail, lista de exercício) & 3 & 1 & 2 & 2,21 & 31 \\
\hline 17 & Treina conteúdos aprendidos, mesmo sabendo que não valem nota & 3 & 1 & 2 & 2,21 & 31 \\
\hline 18 & Faz anotações do que acha importante, quando assiste uma aula & 3 & 2 & 1 & 2,79 & 39 \\
\hline 19 & Estuda em casa mesmo sabendo que não vale nota & 3 & 2 & 1 & 2,57 & 36 \\
\hline 24 & Revisa um conteúdo anterior antes de cada aula & 3 & 1 & 2 & 2,21 & 31 \\
\hline 27 & Faz questões para si mesmo sobre o que estudou & 3 & 1 & 2 & 1,57 & 22 \\
\hline 28 & Faz anotações ao lado do texto & 3 & 1 & 2 & 2,50 & 35 \\
\hline 31 & Destaca as partes importantes de diferentes disciplinas para aprender melhor & 3 & 1 & 2 & 2,50 & 35 \\
\hline 32 & Escreve um rascunho antes da composição final de um texto & 3 & 1 & 2 & 2,50 & 35 \\
\hline 33 & Resume os conteúdos & 3 & 1 & 2 & 2,14 & 30 \\
\hline 34 & Faz anotações sobre os pontos mais importantes do conteúdo & 3 & 2 & 1 & 2,79 & 39 \\
\hline 37 & Procura formular e responder questões por escrito sobre um conteúdo & 3 & 1 & 2 & 2,07 & 29 \\
\hline 39 & Faz leitura sobre o mesmo tema que irá estudar & 3 & 2 & 1 & 2,50 & 35 \\
\hline 40 & Lê em voz alta o que está estudando & 3 & 1 & 2 & 2,21 & 31 \\
\hline
\end{tabular}

Fonte: Elaboração própria.

Em relação as estratégias de aprendizagem cognitivas, foi possível perceber que as mais adotadas pelos licenciandos foram "faz anotações do que acha importante, quando assiste uma aula" e "faz anotações sobre os pontos mais importantes do conteúdo", ambas com média igual a 2,79, com amplitude igual a 1, sendo que nenhum participante marcou a opção "nunca". As duas estratégias são semelhantes, se referindo ao fato de anotar o que se considera importante em determinado assunto, seja durante a aula ou em outro momento de estudo.

As anotações se apresentam como recursos importantes no público estudado, o que sugere a valorização da elaboração e do ensaio do conteúdo a ser aprendido. Essa estratégia é uma das atividades que promovem o sucesso escolar e que ainda merecem ser estimuladas pelos professores neste contexto de aprendizagem, uma vez que é a que mais utilizam (Alliprandini et al., 2014).

Weinstein et al. (2011) enfatizam que, tanto as estratégias de aprendizagem mais simples, como sublinhar, fazer 
anotações, quanto as mais complexas, precisam ser aprendidas, o que supõe ensino específico e treinamento em sua utilização. Em relação as estratégias cognitivas menos utilizadas, identificamos: "cria perguntas e respostas sobre o assunto que está estudando" e "faz questões para si mesmo sobre o que estudou", com 1,79 e 1,57 de média respectivamente. Ambas também possuem similaridade, estando relacionadas com a elaboração de perguntas sobre o conteúdo que está sendo estudado. Martins e Zerbini (2014) também identificaram o baixo uso referente a essa estratégia, e discutem que ela pode ser considerada pelos alunos como uma estratégia que exige uma maior complexidade na sua realização.

O questionamento é uma estratégia de grande valia para a aprendizagem dos estudantes, pois permite a melhoria da aprendizagem dos conteúdos, bem como possibilita que eles desenvolvam diferentes competências, como argumentação, o pensamento crítico e a avaliação (Astolfi, 2008; Martins \& Zerbini, 2014)

Contudo, para que os alunos possam beneficiar de todas as potencialidades pedagógicas do questionamento é necessário valorizar, aquando da sua utilização na sala de aula, determinados princípios pedagógicos e de estratégias que asseguram a sua eficácia e evitar certos procedimentos.

As estratégias de aprendizagem metacognitivas também foram identificadas (Tabela 2), sendo as mais utilizadas pelos alunos: "relê trechos do texto quando encontra uma informação que tem dificuldades para entender", "volta a ler alguns parágrafos ou páginas já lidas quando se distrai", "lê com atenção para ter certeza de que está entendendo" e "realiza atividade ou trabalhos, para entregar, dentro do prazo estabelecido".

Tabela 2. Dados referentes as estratégias de aprendizagem metacognitivas.

\begin{tabular}{|c|l|c|c|c|c|c|}
\hline Item & \multicolumn{1}{|c|}{ Afirmativa } & Pmáx & Pmin & A & MA & PT \\
\hline 1 & Memoriza fórmulas para resolver atividades com maior rapidez & 3 & 1 & 2 & 2,14 & 30 \\
\hline 2 & $\begin{array}{l}\text { Relê trechos do texto quando encontra uma informação que tem dificuldades } \\
\text { para entender }\end{array}$ & 3 & 2 & 1 & 2,86 & 40 \\
\hline 8 & Retoma conteúdos aprendidos na sala de aula & 3 & 2 & 1 & 2,43 & 34 \\
\hline 9 & Pensa sobre um tema antes de começar a escrever & 3 & 1 & 2 & 2,57 & 36 \\
\hline 10 & Volta a ler alguns parágrafos ou páginas já lidas quando se distrai & 3 & 2 & 1 & 2,93 & 41 \\
\hline 11 & $\begin{array}{l}\text { Tenta não se distrair quando o (a) professor (a) está explicando conteúdo } \\
\text { novo }\end{array}$ & 3 & 2 & 1 & 2,71 & 38 \\
\hline 14 & Faz intervalos na leitura quando o texto é muito extenso & 3 & 2 & 1 & 2,64 & 37 \\
\hline 15 & $\begin{array}{l}\text { Memoriza fórmulas, regras gramaticais, termos técnicos que são explicados } \\
\text { em sala }\end{array}$ & 3 & 1 & 2 & 2,07 & 29 \\
\hline 16 & Realiza atividade ou trabalhos, para entregar, dentro do prazo estabelecido & 3 & 2 & 1 & 2,86 & 40 \\
\hline 20 & Relaciona o que está aprendendo com alguma coisa que já sabia & 3 & 1 & 2 & 2,50 & 35 \\
\hline 21 & Organiza e utiliza o tempo livre no trabalho ou em casa para estudar & 3 & 2 & 1 & 2,21 & 31 \\
\hline 22 & Relaciona os conteúdos do curso ao seu dia a dia & 3 & 2 & 1 & 2,21 & 31 \\
\hline 23 & Verifica se atingiu o objetivo que havia estabelecido para o que iria estudar & 3 & 1 & 2 & 2,07 & 29 \\
\hline 25 & Utiliza o tempo livre para fazer atividades do curso & 3 & 1 & 2 & 2,64 & 37 \\
\hline 26 & Persiste na resolução de uma tarefa, mesmo que ela seja difícil & 3 & 2 & 1 & 2,79 & 39 \\
\hline 29 & Antecipa informações do texto quando está compreendendo a leitura & 3 & 1 & 2 & 2,29 & 32 \\
\hline 30 & Verifica se as hipóteses que fez sobre o conteúdo estão certas ou erradas & 3 & 1 & 2 & 2,57 & 36 \\
\hline 35 & Lê com atenção para ter certeza de que está entendendo & 3 & 2 & 1 & 2,86 & 40 \\
\hline 36 & Estuda ou faz atividades do curso diariamente & 3 & 2 & 1 & 2,36 & 33 \\
\hline 38 & Busca solucionar uma dificuldade para aprender determinados assuntos & 3 & 1 & 2 & 2,29 & 32 \\
\hline
\end{tabular}

Fonte: Elaboração própria.

As três primeiras possuem relação entre si, estando voltadas a ler e reler textos com atenção, seja por distração, dificuldade ou para ter certeza de que estão compreendendo aquele conteúdo. Para Dutra e Martins (2012), os alunos possuem muita dificuldade em compreender textos científicos, ainda mais voltados a Química/Física: "a dificuldade em organizar os conteúdos no caderno, em ler e entender os textos usados pelo professor, [...] podem interferir bastante no aprendizado" (p. 
19).

Desse mesmo modo, reler é um dos aspectos associados a comportamentos e pensamentos que o sujeito pode utilizar no decurso da aprendizagem e que influenciam a forma como processa a informação (Figueira, 2006).

A quarta estratégia metacognitiva mais adotada estava relacionada com o compromisso em entregar atividades dentro do prazo estimado pelo professor. Bzuneck (2010) apresenta uma série de estratégias que asseguram um grau motivacional ótimo para aprender, sendo necessário valorizar, dentre outras coisas, a pontualidade nas exigências das tarefas (prazo de entrega).

As estratégias metacognitivas indicadas pelos licenciandos como as menos utilizadas por eles foram "memoriza fórmulas, regras gramaticais, termos técnicos que são explicados em sala" e "verifica se atingiu o objetivo que havia estabelecido para o que iria estudar".

Os resultados de algumas pesquisas vão de encontro com os dados aqui apresentados referentes a essa primeira categoria menos utilizada, como por exemplo, os trabalhos de Biggs (2003) e Galvão et al. (2012) que identificaram que, para ler, os alunos se relacionavam com essa tarefa basicamente de duas formas: focalizando a compreensão do texto como um todo e focalizando a memorização e a reprodução de conteúdos conceituais do texto.

Perceber que os estudantes indicam o baixo uso dessa estratégia pode ser considerado um ponto positivo, uma vez que o uso da memorização nem sempre é viável a uma aprendizagem efetiva. É necessário ter uma visão mais crítica ao usar a memorização durante a aprendizagem.

A segunda estratégia metacognitiva menos utilizada estava relacionada com a verificação por parte do aluno do atingimento ou não do que se havia estabelecido para o seu estudo. De acordo com Zimmerman (2000) e Casiraghi et al. (2020), o uso dessa estratégia oportuniza o pensar sobre as cognições, o comportamento e a própria aprendizagem, o que inclui a autorregulação da aprendizagem, ou seja, envolve tomar consciência sobre objetivos de estudo para organizar e dirigir o próprio processo de aprendizagem. Estudantes autorregulados são metacognitivos, motivados e participantes ativos nos seus processos de aprendizagem, pois dirigem seus próprios esforços para construção de conhecimentos e desenvolvimento de habilidades.

Para Boruchovitch et al. (2017) o uso de diferentes estratégias de aprendizagem por parte do estudante favorece o seu sucesso acadêmico. Desse modo, é de grande valia disponibilizar aos alunos conhecimento das várias estratégias de aprendizagem cognitivas e metacognitivas, deixando claro que essas estratégias devem ser escolhidas por eles com base no autoconhecimento e de acordo com suas características pessoais ou diferenças individuais, uma vez que elas não funcionam da mesma forma para todos.

A universidade é considerada um espaço de aprendizagem e de socialização, favorecendo desenvolvimento cognitivo/afetivo (Bacan et al., 2020). Assim, é importante que os alunos do ensino remoto percebam essa modalidade como algo enriquecedor, mesmo não estando fisicamente em uma sala de aula nem vivenciando presencialmente o ambiente universitário.

Segundo Assunção e Viana (2020), conhecer os estilos e estratégias de aprendizagem dos alunos possibilita ao professor a modificação das estratégias e metodologias de ensino para atingir todos os alunos de forma efetiva. Uma vez que o professor identifica essas diferenças e procura adotar metodologias que atendam aos diversos polos, o aproveitamento tende a melhorar.

Em vista disso, os ambientes virtuais utilizados no ensino remoto devem oferecer ao aluno instrumentos de socialização, como: videoaula, chat, fórum para dúvidas, bem como outros meios didáticos de comunicação e interação com os envolvidos no processo, para que o aluno faça o uso de diferentes estratégias de aprendizagem. 


\section{Considerações Finais}

Conhecer os perfis dos alunos do ensino remoto e das estratégias que eles adotam, amplia as possibilidades de desenvolvimento e aperfeiçoamento das metodologias de ensino nesta modalidade, favorecendo a adaptação e a motivação dos estudantes.

As evidências da pesquisa citadas ao longo do texto apresentam as estratégias de aprendizagem cognitivas mais utilizadas pelos alunos estavam relacionadas com a ação de anotar o que se considera importante em determinado assunto, seja durante a aula ou em outro momento de estudo. Tais aspectos se revelam importantes na aprendizagem, uma vez que estão relacionadas a movimentos cognitivos que podem resultar na qualificação da aprendizagem.

Os alunos apresentaram utilizar mais estratégias metacognitivas, que são aquelas relacionadas ao aprender a aprender, representando aquelas ações às quais os estudantes recorrem quando se recordam de que forma aprenderam melhor um determinado conteúdo. As mais citadas pelos alunos foram aquelas voltadas a ler e reler textos com atenção, tentando superar a dificuldade ou para ter certeza de que estão compreendendo aquele conteúdo.

Nesse contexto, é importante destacar que o processo educacional no ensino remoto não deve ser uma simplificação do realizado na modalidade presencial, uma vez que toda fundamentação teórica necessária ao desenvolvimento de competências e habilidades, o exercício da cidadania e a autonomia para continuar aprendendo é o mesmo para ambas as modalidades de ensino.

Pesquisas que auxiliam no aperfeiçoamento do ensino remoto contribuem para que esta modalidade, mesmo que na realidade atual tenha sido utilizada de maneira emergencial, possa ser vista como uma alternativa educacional disponível, com aplicabilidade atestada. Desse modo, espera-se que a presente pesquisa forneça conhecimentos e discussões acerca dos elementos necessários para que o ensino remoto melhore constantemente sua qualidade e eficácia, bem como fomente outros estudos que buscam compreender como os estudantes estão lhe dando, no contexto pandêmico, com o seu processo de aprendizagem.

\section{Referências}

Alliprandini. P. M. Z., Schiavoni, A., Méllo, D. E. \& Sekitani, J. T. (2014). Estratégias de Aprendizagem Utilizadas por Estudantes na Educação a Distância: Implicações Educacionais. Psicologia da Educação, 38(1), 5-16.

Andrade, R. B. N. M. \& Zerbini, T. (2020). Estilos e Estratégias de Aprendizagem em Educação a Distância: Diferenças e Semelhanças Conceituais. Revista Psicologia: Organizações \& Trabalho, 20(3), 1150-1156.

Assunção, M. T., \& Viana, L. A. F. de C. (2020). Uma Revisão da Literatura sobre os Estilos de Aprendizagem em Cursos Técnicos, Superiores e de Especialização e sobre os Impactos do Ensino Remoto Emergencial. Research, Society and Development, 9(11), e3429119663.

Astolfi, J. P. (2008). Le Questionnement Pédagogique. Economie et Management, 128, 68-73.

Bacan, A. R., Martins, G. H. \& Santos, A. A. A. (2020). Adaptação ao Ensino Superior, Estratégias de Aprendizagem e Motivação de Alunos EaD. Psicologia: Ciência e Profissão, 40, 1-15.

Bannel, R. I., Duarte, R., Carvalho, C., Pischetola, M., Marafon, G. \& Campos, G. H. (2016). Educação no Século XXI: Cognição, Tecnologias e Aprendizagens. Vozes, Editora PUC.

Biggs, J. B. (2003). Teaching for Quality Learning at University. Open University Press.

Boruchovitch, E., Santos, A. A. A., Costa, E. R. Neves, E. R. C., Cruvinel, M., Primi, R. \& Guimarães, S. E. R. (2006). A Construção de uma Escala de Estratégias de Aprendizagem para Alunos do Ensino Fundamental. Psicologia: Teoria e Pesquisa, 22(3), $297-304$.

Boruchovitch, E., Almeida, L. A., \& Miranda, L. M. (2017). Autorregulação da Aprendizagem e Psicologia Positiva: Criando Contextos Educativos Eficazes e Saudáveis. In Boruchovitch, E., Azzi, R. G. \& Soligo, A. (Eds.). Temas em Psicologia Educacional: Contribuições para Formação de Professores. Mercado de Letras.

Boruchovitch, E. \& Gomes, M. A. M. (2019). Aprendizagem Autorregulada: Como Promovê-la no Contexto Educativo? Vozes.

Bzuneck, J. A. Aprendizagem por Processamento da Informação: uma Visão Construtivista. In: Boruchovitch, E. \& Bzuneck, J. A. (Org.). (2010). Aprendizagem: Processos Psicológicos e o Contesto Social na Escola. Vozes, 2010. 
Casiraghi, B., Boruchovitch, E. \& Almeida, L. s. (2020). Crenças de Autoeficácia, Estratégias de Aprendizagem e o Sucesso Acadêmico no Ensino Superior. Revista E-Psi, 9(1), 27-38.

Castro, J. X., Miranda, G. J. \& Leal, E. A. (2016). Estratégias de Aprendizagem dos Estudantes Motivados. Advances in Scientific and Applied Accounting, $9(1), 80-97$

Dutra, G. \& Martins, M. I. (2012). A Recuperação Paralela no Ensino de Física: O que Pensa o Professor? Ensaio: Avaliação e Políticas Públicas em Educação, 20(74), 135-164.

Figueira, A. P. C. (2006). Estratégias Cognitivo/Comportamentais de Aprendizagem: Problemática Conceptual e outras Rubricas. Revista Iberoamericana de Educación, 37(6), 1-21.

Filatro, A. (2018) Como Preparar Conteúdos para EAD. Saraiva.

Galvão, A., Câmara, J. \& Jordão, M. (2012). Estratégias de Aprendizagem: Reflexões sobre Universitários. Revista Brasileira de Estudos Pedagógicos, 93(235), 627-644.

Hargreaves, A. (2004). O Ensino na Sociedade do Conhecimento: Educação na Era da Insegurança. Artmed.

Horowitz, F. D., Darling-Hammond, L., Bransford, J., Comer, J., Rosebrock, K., Austin, K. \& Rust, F. Formação de Professores em Práticas Apropriadas para o Desenvolvimento. In: Darling-Hammond, L. \& Bransford, J. (Orgs.). (2019). Preparando os Professores para um Mundo em Transformação. Penso.

Huot, R. (2002). Métodos Quantitativos para as Ciências Humanas. Instituto Piaget.

Martins, L. B. \& Zerbini, T. (2014). Escala de Estratégias de Aprendizagem: Evidências de Validade em Contexto Universitário Híbrido. Psico-USF, 19(2), 317-328.

Massoni, N. T. (2016). Projetos de Pesquisa em Educação: Importância, Elaboração e Cuidados. In: Massoni, N. T. \& Moreira, M. A. Pesquisa Qualitativa em Educação em Ciências: Projetos, Entrevistas, Questionários, Teoria Fundamentada, Redação Científica. Livraria da Física.

Moreira, J. A., Henriques, S. \& Barros, D. (2020). Transitando de um Ensino Remoto Emergencial para uma Educação Digital em Rede, em Tempos de Pandemia. Dialogia, 34, 351-364.

Oliveira, K. L., Boruchovitch, E. \& Santos, A. A. A. (2009). Estratégias de Aprendizagem e Desempenho Acadêmico: Evidências de Validade. Psicologia: Teoria e Pesquisa, 24(4), 531-536.

Oliveira, K. L., Boruchovitch, E. \& Santos, A. A. A. (2010). Escala de Avaliação das Estratégias de Aprendizagem para o Ensino Fundamental (EAVAP-EF). Casa do Psicólogo.

Pilleti, C. (2004). Didática Geral. Ática.

Rosa, C. T. W. (2011). A Metacognição e as Atividades Experimentais no Ensino de Física. Tese (Doutorado em Educação Científica e Tecnológica) Universidade Federal de Santa Catarina, Florianópolis/SC.

Sampieri, R. H., Collado, C. F., \& Lucio, M. P. B. (2013). Metodologia da Pesquisa. Penso.

Suart, R. C., Marcondes, M. E. R. \& Lamas, M. F. P. (2010). A Estratégia "Laboratório Aberto" para a Construção do Conceito de Temperatura de Ebulição e a Manifestação de Habilidades Cognitivas. Química Nova na Escola, 32(3), 200-207.

Vieira, H. V. P., Tamiasso-Martinhon, P., Simões, A. L., Rocha, A. S. \& Souza, C. (2019). Perspectivas do Uso de Aplicativos de Celular como Ferramenta Pedagógica para o Ensino de Química. Revista Debates em Ensino de Química, 5(1), 125-138.

Weinstein, C. E., Acee, T. W., \& Jung, J. (2011). Self-Regulation and Learning Strategies. New Directions for Teaching \& Learning, 126, 45-53.

Zerbini, T. \& Abbad, G. (2008) Estratégias de Aprendizagem em Curso a Distância: Validação de uma Escala. Psico-USF, 13(2), 177-187.

Zimmerman, B. J. (2000). Attaining Self-Regulation: A Social Cognitive Perspective. In Boekarts, M. P. R. Pintrich, \& Zeidner, M. (Eds.). Handbook of SelfRegulation. Academic Press. 